\title{
Blood Protein Concentrations in the First Two Postnatal Weeks That Predict Bronchopulmonary Dysplasia Among Infants Born Before the 28th Week of Gestation
}

\author{
CARL BOSE, MATTHEW LAUGHON, ELIZABETH N. ALLRED, LINDA J. VAN MARTER, T. MICHAEL O'SHEA, \\ RICHARD A. EHRENKRANZ, RAINA FICHOROVA, AND ALAN LEVITON; FOR THE ELGAN STUDY INVESTIGATORS \\ Department of Pediatrics [C.B., M.L.], School of Medicine, University of North Carolina, Chapel Hill, North Carolina 27599; Department \\ of Biostatistcis [E.N.A.], Harvard School of Public Health, Boston, Massachusetts 02215; Departments of Pediatrics [L.J.M.], Obstetrics, \\ Gynecology and Reproductive Biology [R.F.], and Neurology [A.L.], Harvard Medical School, Boston, Massachusetts 02215; Department \\ of Pediatrics [T.M.O.], Wake Forest University School of Medicine, Winston-Salem, North Carolina 27157; Department of Pediatrics
}

[R.A.E.], Yale School of Medicine, New Haven, Connecticut 06510

\begin{abstract}
Lung inflammation contributes to the pathogenesis of bronchopulmonary dysplasia (BPD) and may be accompanied by a systematic inflammatory response. The objective of this study was to investigate the role of systemic inflammation in the development of BPD in a cohort of extremely low GA newborns (ELGANs) by examining the relationships between inflammation-associated proteins in neonatal blood samples and pulmonary outcomes. Proteins were measured in blood specimens collected on postnatal d 1-3, 5-8, and 12-15 from 932 ELGANs. Increased risk of BPD was associated with elevated blood concentrations of a variety of proinflammatory cytokines, adhesion molecules, and proteases. Reduced risk was prominently associated with increased concentrations of one chemokine, RANTES. Elevations of inflammatory proteins associated with BPD risk occurred during the first days after birth and inflammation intensified thereafter. Therefore, exposures that promote inflammation after the first postnatal days may be more critical in the pathogenesis of BPD. Fetal growth restriction, a known BPD risk factor, was not accompanied by proteins elevations and therefore does not seem to be mediated by systemic inflammation. By contrast, mechanical ventilation altered protein levels and may be associated with systemic inflammation. (Pediatr Res 69: 347-353, 2011)
\end{abstract}

$\mathrm{S}^{\mathrm{u}}$ upport for the claim that early postnatal inflammation contributes to the pathogenesis of bronchopulmonary dysplasia (BPD) comes from several sources. First, the number of inflammatory cells in the airways of preterm infants increases during acute lung disease, and this increase persists in infants who develop BPD $(1,2)$. Second, preterm infants who develop BPD are more likely than their peers to have elevated concentrations of inflammatory mediators in their tracheal fluid $(3,4)$. Finally, in animal models, exposures associated with BPD (e.g. mechanical ventilation and increased ambient oxygen) increase gene expression of inflam-

Received September 2, 2010; accepted October 21, 2010.

Correspondence: Carl Bose, M.D., Division of Neonatal-Perinatal Medicine, UNC Hospital, CB\#7596, Chapel Hill, NC 27599-7596; e-mail: cbose@med.unc.edu

Supported by a cooperative agreement with The National Institute of Neurological Disorders and Stroke (NINDS; 5U01NS040069-04). In addition, C.B. was partially supported by the Thrasher Research Fund. mation-associated proteins and increase levels of these proteins in the lung $(5,6)$.

The finding of altered levels of inflammation-associated proteins in the blood of infants who develop BPD $(7,8)$ indicates that BPD may be associated with a systemic inflammatory response, but it is not clear whether these proteins originate in the lung or elsewhere. In either case, measurement of biomarkers of inflammation in the blood may provide insight about the role of inflammation in the development of BPD.

The objective of this study was to investigate the role of inflammation in the development of BPD in a cohort of extremely low GA newborns (ELGANs) by examining the relationships between biomarkers in neonatal blood samples and pulmonary outcomes. We measured biomarker levels in serial blood specimens collected soon after birth and during the subsequent $2 \mathrm{wk}$. Our goal was to investigate the role of inflammation before and after neonatal exposures that might promote inflammation. Of particular interest were changes in levels of biomarkers among infants with factors known to increase the likelihood of BPD, including fetal growth restriction and mechanical ventilation. Identifying relationships between these risk factors and specific biomarkers might suggest mechanisms through which these exposures impart risk. We examined these relationships separately in infants with mild/ moderate and severe BPD to evaluate the possibility that the pathophysiology and the role of inflammation might be distinct in these two entities.

\section{METHODS}

This study was a component of the ELGAN Study, a multicenter study designed to identify characteristics and exposures that increase the risk of neurologic disorders in ELGANs (9). During the years 2002-2004, women delivering before 28 -wk gestation at one of 14 institutions were enrolled in the study. The study was approved by the individual institutional review boards, and informed consent was obtained from participating mothers. The original consent and approval included the provision for secondary analyses of deidentified data.

Abbreviations: BPD, bronchopulmonary dysplasia; ELGAN, extremely low GA newborn; PMA, postmenstrual age; $\mathbf{R}$, receptor 
Our study cohort consisted of all infants in the ELGAN Study who survived to 36-wk postmenstrual age (PMA) for whom protein measurements were available in one or more blood specimens obtained during the first two postnatal weeks. Because of financial constraints, measurement of proteins was limited to specimens from infants who survived until the 24-mo adjusted age and for whom all elements of the follow-up evaluation at 24 mo were completed, including developmental testing, neurologic examination, and measurement of head circumference. A full description of the methods of the ELGAN Study is provided elsewhere (9). Here, we focus on those most relevant to this report.

Maternal, pregnancy, and newborn variables. Selected characteristics of the pregnancy, intrapartum, and newborn period were recorded. The GA estimates were based on a hierarchy of the quality of available information that included (in order of priority) the following: 1) dates of embryo retrieval or intrauterine insemination or fetal ultrasound before the 14th week $(62 \%)$, 2) fetal ultrasound at 14 or more weeks $(29 \%)$, 3) last menstrual period without fetal ultrasound (7\%), and 4) GA recorded in the log of the NICU (1\%). The birth weight birth weight $Z$ score for each infant was calculated using a standard data set (10).

Data describing the highest level of respiratory support, categorized as no support, increased ambient oxygen, nasal continuous positive airway pressure, conventional mechanical ventilation, or high-frequency ventilation, were collected daily during the first week and on d 7, 14, 21, and 28, and at 36-wk PMA. We did not record the specific devices used.

BPD was defined as oxygen therapy at 36 wk PMA. Decisions to administer supplemental oxygen were made by infants' clinical providers and were not based on a uniform threshold of blood oxygenation. We subdivided these infants into those who were not ventilator dependent (mild/moderate BPD) and those who were ventilator dependent (severe BPD).

Placenta morphology. Samples were collected from placentas for histologic examination and were processed for morphologic assessment as part of routine clinical care. Details describing the criteria for each histologic lesion are presented elsewhere (11).

Blood collection and protein measurements. Blood was collected on filter paper (Schleicher \& Schuell 903). The times of collection are designated as d 1,7 , and 14 and were defined by the following ranges of postnatal age at collection: $\mathrm{d} 1$ range $=\mathrm{d} 1-3 ; \mathrm{d} 7$ range $=\mathrm{d} 5-8$; and $\mathrm{d} 14$ range $=\mathrm{d} 12-15$. Dried blood spots were stored at $-70^{\circ} \mathrm{C}$ in sealed bags with desiccant until processed.

Analyses of proteins were performed in the Laboratory of Genital Tract Biology, Brigham \& Women's Hospital. For protein elution, 12-mm punched biopsies of the frozen blood spots were submerged in 300- $\mu$ L PBS containing $0.1 \%$ Triton $\times 100$ (Sigma Chemical Co.-Aldrich, St. Louis, MO) and 0.03\% Tween-20 (Fisher, Hampton, NH), vortexed for $30 \mathrm{~s}$, and incubated on a shaker for $1 \mathrm{~h}$ at $4^{\circ} \mathrm{C}$. The buffer and biopsy were then transferred over the filter of a SpinX tube (Corning Fisher), centrifuged at $2000 \times g$ followed by collection of the filtered eluted blood. An additional wash of the punch was performed in $100 \mu \mathrm{L}$ for a final elution volume of $400 \mu \mathrm{L}$.

Proteins were measured in duplicate using the Meso Scale Discovery multiplex platform and Sector Imager 2400 (MSD, Gaithersburg, MD). This electrochemiluminescence system has been validated by comparisons with traditional ELISA (12). The MSD Discovery Workbench Software was used to convert relative luminescent units into protein concentrations using interpolation from several log calibrator curves. Split quality control blood pools tested on each plate showed interassay variation of $10-20 \%$ for each protein. The total protein concentration in each eluted sample was determined by BCA assay (Thermo Scientific, Rockford, IL) using a multilabel Victor 2 counter (Perkin Elmer, Boston, MA), and the measurement of each analyte was normalized to milligrams total protein.

We measured the following 25 proteins: IL-1B, IL-6, IL-6R ( $\mathrm{R}=$ receptor), TNF- $\alpha$, TNF-R1, TNF-R2, IL-8 (CXCL8; IL-8), MCP-1 (CCL2; monocyte chemotactic protein-1), MCP-4 (CCL13; monocyte chemoattractant protein-4), MIP-1ß (CCL4; macrophage inflammatory protein-1ß), RANTES (CCL5; regulated upon activation, normal $\mathrm{T}$ cell expressed, and secreted), I-TAC (CXCL11; interferon-inducible $\mathrm{T}$ cell alpha-chemoattractant), ICAM-1 (CD54), ICAM-3 (CD50), VCAM-1 (CD106; vascular cell adhesion molecule-1), E-selectin (CD62E; E-SEL), MMP-1 (matrix metalloproteinase1), MMP-9 (matrix metalloproteinase-9), CRP (C-reactive protein), SAA (serum amyloid A), MPO (myeloperoxidase), VEGF, VEGF-R1, VEGF-R2, and IGFBP-1 (insulin growth factor binding protein-1).

Data analysis. We evaluated the generalized null hypothesis that the risks of mild/moderate and severe BPD are not associated with blood protein concentrations in the highest quartile for GA and the day the blood was collected. We began our analyses by exploring the frequency of each entity in the four quartiles of the concentration of each protein. Linear trends were rare so our final analyses are limited to comparisons of infants whose protein concentration was in the highest quartile with infants whose protein concen- tration was in the lower three quartiles. An association of risk of each BPD entity with a protein concentration in the highest quartile was defined by an OR and the 99\% CI. We selected this CI rather than the conventional 95\% CI because we wanted to modify our analyses for multiple comparisons, while not appreciably increasing the risk of a type 2 error (13).

Because the two BPD entities are mutually exclusive and each is compared with the same referent group (infants who did not develop BPD), we used multinomial logistic regression (13) and controlled for GA (23-24, 25-26, and $27 \mathrm{wk})$ to evaluate protein-BPD relationships at each time point $(\mathrm{d} 1,7$, and 14).

We evaluated the antecedents of lung injury in the order they occur by creating time-oriented logistic regression models that first considered endogenous characteristics of the infant (e.g. GA and birth weight $Z$ score). Then, we sequentially added information conveyed by variables for an elevated concentration of each protein in the temporal order the blood was collected. In one model, mechanical ventilation was added at $\mathrm{d} 7$ if the infant was being treated with any form of mechanical ventilation on that day. In these timeoriented models, significant variables $(p<0.01)$ are retained for inclusion in the model with information from the next time interval or epoch. The final model included variables from each of the three specimen collection intervals. We used a step-down procedure seeking a parsimonious solution without interaction terms.

\section{RESULTS}

During the study period, 1249 mothers of 1506 infants consented to participate in the ELGAN Study. Among these infants, 255 died before 36 wk PMA and 51 died between 36 wk PMA and 2 y adjusted age (Fig. 1). An additional 258 were excluded from our study because either follow-up evaluation was incomplete or blood specimens were not available. Our cohort included the remaining 932 infants. Frequency of maternal, pregnancy, and newborn features within each category are listed in Table 1. Four hundred infants (43\%) were oxygen dependent but not ventilator dependent at 36 wk PMA, and an additional 86 infants (9\%) were both oxygen dependent and ventilator dependent.

Among the 258 infants who survived to 24 mo adjusted age, but for whom measurements of proteins were not available, the incidence of mild/moderate BPD was $39 \%$, and the incidence of severe BPD was 5\%. Among the 51 infants who died between 36 wk PMA and 24 mo adjusted age, the incidence of mild/moderate BPD and severe BPD was 39 and $47 \%$, respectively.

Protein measurements were available for 855 infants on d 1, 860 infants on d 7, and 781 infants on d 14. Among all infants,

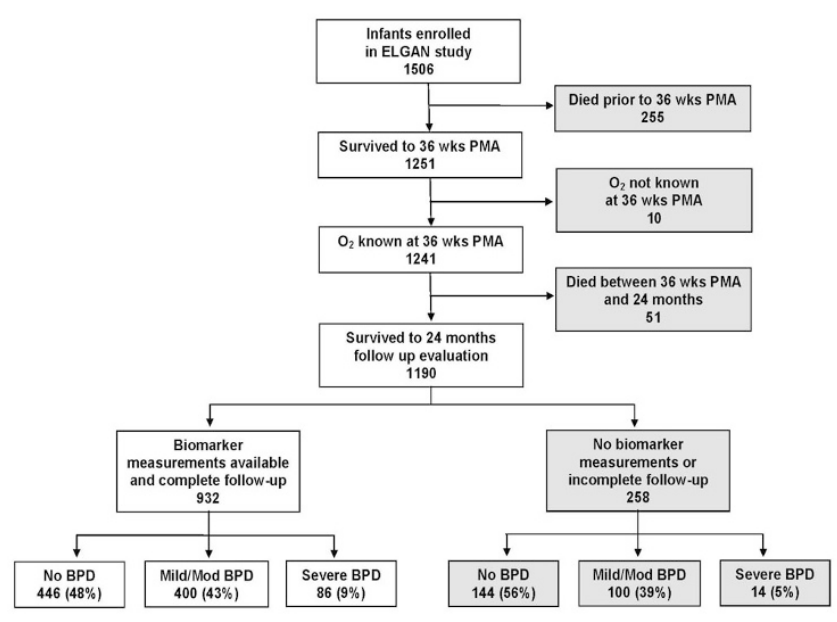

Figure 1. Derivation of the study population. 
Table 1. Antenatal, delivery, and neonatal characteristics of infants classified by their oxygen and ventilator dependency at 36 wk PMA

\begin{tabular}{|c|c|c|c|c|}
\hline \multirow[b]{2}{*}{ Characteristics } & \multicolumn{3}{|c|}{$\begin{array}{c}\text { Bronchopulmonary } \\
\text { dysplasia* }\end{array}$} & \multirow[b]{2}{*}{$\mathrm{N}$} \\
\hline & None & $\begin{array}{c}\text { Mild/ } \\
\text { moderate } \dagger\end{array}$ & Severe $\neq$ & \\
\hline \multicolumn{5}{|l|}{ Maternal race } \\
\hline White & 59 & 64 & 62 & 561 \\
\hline Black & 28 & 26 & 32 & 251 \\
\hline \multicolumn{5}{|c|}{ Antenatal corticosteroid course } \\
\hline Complete & 67 & 62 & 60 & 599 \\
\hline Partial & 22 & 28 & 25 & 233 \\
\hline \multicolumn{5}{|l|}{ Cesarean delivery } \\
\hline Yes & 67 & 66 & 71 & 621 \\
\hline \multicolumn{5}{|l|}{ GA (wk) } \\
\hline $23-24$ & 10 & 30 & 31 & 188 \\
\hline $25-26$ & 44 & 49 & 50 & 433 \\
\hline \multicolumn{5}{|l|}{ Birth weight (g) } \\
\hline$\leq 750$ & 19 & 51 & 61 & 342 \\
\hline $751-1000$ & 52 & 38 & 31 & 409 \\
\hline \multicolumn{5}{|l|}{ Birth weight $Z$ score } \\
\hline$<-2$ & 3 & 7 & 13 & 50 \\
\hline$\geq-2,<-1$ & 7 & 18 & 51 & 121 \\
\hline \multicolumn{5}{|l|}{ Sex } \\
\hline Male & 51 & 55 & 49 & 490 \\
\hline \multicolumn{5}{|l|}{ Fetuses } \\
\hline Multiple & 35 & 32 & 36 & 316 \\
\hline \multicolumn{5}{|l|}{ Chorioamnionitis } \\
\hline Yes & 36 & 37 & 29 & 305 \\
\hline \multicolumn{5}{|l|}{ Funisitis } \\
\hline Yes & 17 & 18 & 11 & 142 \\
\hline Maximum number & 446 & 400 & 86 & 932 \\
\hline
\end{tabular}

* Numbers in cells are column percentages.

$\dagger$ Oxygen dependent but not ventilator dependent at 36 wk PMA.

$\$$ Oxygen and ventilator dependent at 36 wk PMA.

concentrations of most proteins tended to increase with advancing postnatal age (data not shown). In multinomial analysis, infants who developed mild/moderate BPD, compared with infants with no BPD, had elevated d 1 concentrations of TNF- $\alpha$, elevated d 7 concentrations of TNF-R2, MCP-1, and ICAM-1, and elevated d 14 concentrations of IL-1b, IL-6, TNF- $\alpha$, TNF-R1, IL-8, MCP-1, ICAM-1, and MMP-9 (Table 2). In contrast, these infants had concentrations of RANTES that were significantly reduced on $\mathrm{d} 7$ and 14 and MIP-1B on $\mathrm{d} 7$.

Compared with infants with no BPD, infants who developed severe BPD did not have an appreciable inflammation signal in their d 1 blood (Table 2). Rather, the signal was first evident in $\mathrm{d} 7$ blood with elevated concentrations of TNF-R2, MCP-1, and ICAM-1. The inflammation signal was even more apparent on d 14 with elevated concentrations of IL-6, TNF $\alpha$, TNF-R1, MCP-1, ICAM-1, E-selectin1, and MMP-9. Severe BPD was also preceded by reduced concentrations of RANTES (d 7 and 14) and MMP-1 (d 1 and 14).

We created three time-oriented risk models for the two BPD severity levels. All included GA in three strata (23-24, 25-26, and $27 \mathrm{wk}$ ); the second also included birth weight $Z$ score $<$ -1 and the third included birth weight $Z$ score $<-1$ and mechanical ventilation on $d 7$. These models were created to identify the influence of fetal growth restriction and mechan- ical ventilation on blood protein concentrations. Acquired sepsis and receipt of postnatal steroids did not predict BPD nor did their addition change the proteins selected in the modeling process.

Time-oriented risk model with $\boldsymbol{G A}$ only. In this model, increased risk of mild/moderate BPD was associated with an elevated concentration of TNF- $\alpha$ on d 1(Table 3 ). An elevated concentration of TNF-R2 on d 7 was also associated with increased risk, whereas infants who had elevated concentrations of RANTES and MIP-1ß on $\mathrm{d} 7$ were at reduced risk. The d 7 RANTES risk information was diminished by the addition of d 14 RANTES information. IL-1ß and ICAM-1 were the $2 \mathrm{~d} 14$ proteins whose elevated concentrations provided supplemental information about an increased risk of the mild/moderate BPD.

Elevated concentrations of VCAM-1 on d 1 were associated with an increased risk of severe BPD, whereas elevated concentrations of MMP-1 on d 1 were associated with decreased risk. Both of these proteins continued to convey information about risk even when the information conveyed by $\mathrm{d} 7$ and $\mathrm{d}$ 14 proteins was added to the model. Elevated concentrations of d 7 ICAM-1 and MCP-1 were associated with increased risk, whereas increased concentrations of RANTES were associated with reduced risk. The risk information provided by the RANTES d 7 concentrations was diminished by the addition of d 14 RANTES concentrations to the model. Similarly, the association with $\mathrm{d} 7$ ICAM-1 concentrations was attenuated when the d 14 ICAM-3 concentrations were added to the model.

Time-oriented risk model with $G A$ and birth weight $\mathrm{Z}$ score $<-1$. Birth weight $Z$ score $<-1$ predicted both mild/moderate and severe BPD in all epochs even after adjustment for GA and protein levels (Table 4). The inclusion of birth weight $Z$ score resulted in relatively minor changes in the d 1 protein levels that had been identified as predictors in the model that excluded birth weight $Z$ score. TNF- $\alpha$ remained in the model, as did RANTES on d 14, but RANTES on $\mathrm{d} 7 \mathrm{did}$ not. The predictors of severe BPD changed minimally. ICAM-1 was not added on $\mathrm{d} 7$ as it had been in the previous model, whereas ICAM-1 replaced ICAM-3 on d 14.

Time-oriented risk model with GA, birth weight $\mathrm{Z}$ score $<-1$, and mechanical ventilation on $d 7$. Mechanical ventilation on $\mathrm{d} 7$ was a predictor of both mild/moderate and severe BPD and was a stronger predictor of severe BPD than all other risk factors, including protein levels (Table 5). Adjusting for mechanical ventilation on $\mathrm{d} 7$ altered the model for mild/moderate BPD by dropping ICAM-1 on both $\mathrm{d} 7$ and 14 and RANTES on d 14. Adjusting for mechanical ventilation altered the model for severe BPD by eliminating RANTES on both $\mathrm{d} 7$ and 14 and MCP-2 on d 7. ICAM-1 on d 14 was replaced by ICAM-3.

\section{DISCUSSION}

Our study demonstrates that the risk of BPD among infants born before the 28th wk of gestation is associated with elevated blood concentrations of a variety of proteins integral to inflammation. These include proinflammatory cytokines, 
Table 2. ORs (99\% CIs) from a multinomial analysis for mild/moderate BPD and severe BPD associated with highest quartile of the distribution of inflammation-associated proteins collected at specific postnatal ages and adjusted for GA (23-24, 25-26, and 27 wk)

\begin{tabular}{|c|c|c|c|c|c|c|}
\hline & \multicolumn{3}{|c|}{ Mild/moderate BPD } & \multicolumn{3}{|c|}{ Severe BPD } \\
\hline & Day 1 & Day 7 & Day 14 & Day 1 & Day 7 & Day 14 \\
\hline CRP & $1.5(0.96,2.3)$ & $1.3(0.9,2.0)$ & $1.4(0.9,2.2)$ & $1.3(0.6,2.7)$ & $2.0(0.99,3.9)$ & $1.4(0.6,3.0)$ \\
\hline SAA & $1.6(1.00,2.4)$ & $0.8(0.5,1.2)$ & $1.1(0.7,1.8)$ & $0.9(0.4,2.1)$ & $0.7(0.3,1.5)$ & $0.8(0.3,1.8)$ \\
\hline MPO & $1.0(0.7,1.6)$ & $1.1(0.7,1.6)$ & $1.5(0.95,2.3)$ & $1.4(0.7,2.7)$ & $1.0(0.5,2.0)$ & $1.3(0.6,2.7)$ \\
\hline IL- $1 \beta$ & $1.2(0.8,1.9)$ & $1.2(0.8,1.8)$ & $2.4(1.5,3.7)$ & $0.8(0.4,1.7)$ & $1.4(0.7,2.8)$ & $1.8(0.9,3.8)$ \\
\hline IL-6 & $1.0(0.7,1.6)$ & $0.9(0.6,1.4)$ & $1.8(1.1,2.8)$ & $0.7(0.3,1.5)$ & $1.3(0.7,2.6)$ & $2.0(0.96,4.1)$ \\
\hline IL-6R & $1.0(0.7,1.6)$ & $1.4(0.9,2.2)$ & $1.3(0.9,2.1)$ & $1.2(0.6,2.4)$ & $1.6(0.9,3.2)$ & $1.0(0.5,2.2)$ \\
\hline TNF- $\alpha$ & $1.8(1.2,2.7)$ & $1.3(0.9,2.0)$ & $1.9(1.2,3.0)$ & $1.9(0.96,3.9)$ & $1.6(0.8,3.2)$ & $2.3(1.1,4.8)$ \\
\hline TNF-R1 & $1.1(0.7,1.7)$ & $1.4(0.9,2.2)$ & $2.1(1.3,3.4)$ & $1.3(0.6,2.7)$ & $1.9(0.96,3.8)$ & $3.2(1.5,6.5)$ \\
\hline TNF-R2 & $1.3(0.8,2.0)$ & $1.6(1.02,2.5)$ & $1.4(0.9,2.2)$ & $1.7(0.9,3.5)$ & $2.1(1.1,4.2)$ & $1.9(0.9,3.9)$ \\
\hline IL-8 (CXCL8) & $1.5(0.97,2.3)$ & $1.2(0.8,1.9)$ & $2.2(1.4,3.5)$ & $1.3(0.6,2.7)$ & $1.4(0.7,2.8)$ & $2.1(0.98,4.4)$ \\
\hline MCP-1 (CCL2) & $1.3(0.9,2.0)$ & $1.6(1.04,2.5)$ & $1.7(1.1,2.7)$ & $1.3(0.6,2.6)$ & $3.0(1.5,5.9)$ & $2.7(1.3,5.6)$ \\
\hline MCP-4 (CCL13) & $1.2(0.6,2.6)$ & $1.4(0.9,2.2)$ & $1.1(0.7,1.7)$ & $0.9(0.4,2.0)$ & $1.7(0.9,3.4)$ & $1.0(0.4,2.1)$ \\
\hline MIP-1B (CCL4) & $1.0(0.7,1.6)$ & $0.6(0.4,0.95)$ & $0.8(0.5,1.3)$ & $0.7(0.3,1.6)$ & $1.7(0.3,1.4)$ & $0.6(0.3,1.4)$ \\
\hline RANTES (CCL5) & $0.8(0.5,1.2)$ & $0.6(0.4,0.95)$ & $0.5(0.3,0.8)$ & $0.5(0.2,1.2)$ & $0.3(0.1,0.7)$ & $0.3(0.1,0.8)$ \\
\hline I-TAC (CXCL11) & $1.3(0.8,2.0)$ & $1.1(0.7,1.7)$ & $1.0(0.6,1.6)$ & $1.2(0.6,2.5)$ & $1.4(0.7,2.8)$ & $1.1(0.5,2.3)$ \\
\hline ICAM-1 (CD54) & $1.4(0.9,2.2)$ & $1.7(1.1,2.7)$ & $3.5(2.1,5.8)$ & $1.6(0.8,3.4)$ & $2.5(1.3,5.0)$ & $9.1(4.3,19)$ \\
\hline ICAM-3 (CD50) & $0.9(0.6,1.3)$ & $1.0(0.6,1.5)$ & $1.5(0.9,2.3)$ & $1.0(0.5,2.1)$ & $0.6(0.3,1.4)$ & $1.5(0.7,3.2)$ \\
\hline VCAM-1 (CD106) & $1.5(0.95,2.3)$ & $1.0(0.6,1.5)$ & $1.2(0.8,1.9)$ & $1.7(0.8,3.4)$ & $1.2(0.6,2.4)$ & $1.9(0.9,3.9)$ \\
\hline E-SEL (CL62E) & $1.4(0.9,2.1)$ & $1.4(0.9,2.1)$ & $1.4(0.9,2.3)$ & $0.8(0.4,1.8)$ & $1.4(0.7,2.8)$ & $2.5(1.2,5.1)$ \\
\hline MMP-1 & $0.8(0.5,1.3)$ & $0.7(0.5,1.1)$ & $0.7(0.4,1.1)$ & $0.4(0.2,0.9)$ & $0.5(0.2,1.2)$ & $0.3(0.1,0.8)$ \\
\hline MMP-9 & $0.9(0.6,1.5)$ & $1.3(0.8,2.0)$ & $2.0(1.2,3.2)$ & $1.2(0.6,2.5)$ & $0.6(0.3,1.5)$ & $2.1(1.02,4.5)$ \\
\hline VEGF & $1.0(0.6,1.5)$ & $1.0(0.7,1.6)$ & $1.0(0.6,1.6)$ & $0.8(0.4,1.8)$ & $0.4(0.2,1.04)$ & $0.6(0.3,1.4)$ \\
\hline VEGF-R1 & $1.4(0.9,2.2)$ & $1.3(0.8,1.9)$ & $1.3(0.8,2.0)$ & $0.9(0.4,2.0)$ & $1.1(0.6,2.2)$ & $1.3(0.6,2.6)$ \\
\hline VEGF-R2 & $1.0(0.7,1.5)$ & $1.1(0.7,1.7)$ & $1.3(0.9,2.0)$ & $1.0(0.5,2.0)$ & $0.9(0.5,1.9)$ & $1.7(0.8,3.5)$ \\
\hline IGFBP-1 & $1.5(0.95,2.3)$ & $1.3(0.8,2.1)$ & $1.3(0.9,2.1)$ & $1.2(0.6,2.5)$ & $1.4(0.7,2.9)$ & $2.0(0.95,4.1)$ \\
\hline N BPD & 374 & 363 & 342 & 77 & 80 & 71 \\
\hline $\mathrm{N}$ total & 855 & 860 & 781 & 855 & 860 & 781 \\
\hline
\end{tabular}

The referent group consists of infants who had no BPD.

adhesion molecules, and proteases. Reduced risk is prominently associated with increased concentrations of one chemokine, RANTES. These findings suggest that an inflammatory process involving a variety of mediators is critical in the development of BPD. Elevations of inflammatory proteins associated with BPD risk occur during the first days after birth. However, inflammation intensifies thereafter. Therefore, exposures that promote inflammation after the first postnatal days may be more critical in the development of BPD.

Our observations are in general agreement with previous reports. For example, in a cohort of ELGANs, death or BPD at 36 wk PMA was predicted by elevated blood concentrations of a variety of cytokines (7). The notable similarities to our findings were the increased risk associated with high levels of $\mathrm{TNF} \alpha$ and decreased risk with high levels of RANTES. In contrast to that study, we did not find a distinct pattern of decreased concentrations of proteins that the authors associated with adaptive (as opposed to innate) immunity in infants who developed BPD, with the exception of RANTES. Our choice of proteins might have masked these trends. For example, we did not measure IFN $\gamma$ or TNFß, cytokines associated with innate immunity, or IL-10, a cytokine associated with adaptive immunity (14). A more important difference between these studies might be our exclusion of infants who died before 36 wk PMA. In some studies of BPD, death before 36 wk PMA is included as an outcome measure because it competes with BPD. However, because a relatively small proportion of infants die from lung disease, and some die of multisystem organ failure or sepsis, processes with a strong systemic inflammatory component, we believe that it is more appropriate to exclude infants who die before $36 \mathrm{wk}$ PMA when investigating the role of inflammation in lung disease.

We observed a doubling of risk of less severe BPD among infants with concentrations of TNF $\alpha$ in the highest quartile on the first postnatal day. A similar association was not seen in another cohort of premature infants (15). Several important differences in study design might explain this lack of agreement. For example, that study included infants born before the $33 \mathrm{rd}$ wk of gestation, whereas our study did not include any infant born between wk 28-32. The differences may also have resulted from different methods to account for the potential contribution of chorioamnionitis. We did not adjust for the presence of chorioamnionitis in our regression models because in a previous study of BPD risk in this cohort, we did not identify chorioamnionitis as a risk factor (16). In addition, that study measured concentrations in cord blood only, while the specimens in our study were obtained postnatally. In both humans and animals, proinflammatory cytokines appear in the lungs soon after early neonatal exposures (e.g. mechanical ventilation) that promote inflammation $(17,18)$, raising the possibility that the early elevations in $\mathrm{TNF} \alpha$ in our infants are a consequence of postnatal exposures.

The chemokine RANTES was one of the few proteins associated with reduced risk of both mild/moderate and severe BPD. As a chemotactic agent, it attracts inflammatory cells to the site of infection or injury (19). Therefore, one might 
Table 3. ORs (and $99 \%$ CIs) of mild/moderate BPD and severe $B P D$ (vs no BPD) associated with a concentration of the protein on the left in the highest quartile for GA and day blood was obtained relative to the risk among infants whose concentration was in the lower three quartiles

\begin{tabular}{|c|c|c|c|}
\hline Protein & Day 1 & Days 1 and 7 & Days 1,7 , and 14 \\
\hline \multicolumn{4}{|c|}{ Model for mild/moderate BPD } \\
\hline \multicolumn{4}{|l|}{ Day 1} \\
\hline GA $23-24$ & $6.4(3.7,12)$ & $7.8(4.1,15)$ & $9.2(4.5,19)$ \\
\hline GA $25-26$ & $2.5(1.6,3.9)$ & $2.7(1.6,4.3)$ & $2.9(1.7,5.0)$ \\
\hline TNF- $\alpha$ & $1.8(1.2,2.8)$ & $2.1(1.3,3.3)$ & $2.0(1.2,3.5)$ \\
\hline \multicolumn{4}{|l|}{ Day 7} \\
\hline RANTES & & $0.6(0.4,0.9)$ & $0.7(0.4,1.2)$ \\
\hline TNF-R2 & & $2.1(1.3,3.5)$ & $1.8(1.03,3.3)$ \\
\hline MIP- $1 \beta$ & & $0.5(0.3,0.8)$ & $0.5(0.3,0.9)$ \\
\hline \multicolumn{4}{|l|}{ Day 14} \\
\hline IL-1 $\beta$ & & & $2.0(1.2,3.4)$ \\
\hline RANTES & & & $0.5(0.3,0.9)$ \\
\hline ICAM-1 & & & $2.8(1.6,5.0)$ \\
\hline \multicolumn{4}{|c|}{ Model for severe BPD } \\
\hline \multicolumn{4}{|c|}{ Day 1} \\
\hline GA $23-24$ & $9.0(3.3,24)$ & $11(3.5,32)$ & $15(3.8,60)$ \\
\hline GA $25-26$ & $3.0(1.3,7.2)$ & $3.0(1.2,7.7)$ & $5.1(1.6,16)$ \\
\hline VCAM-1 & $2.5(1.1,5.4)$ & $3.2(1.3,7.6)$ & $3.8(1.3,11)$ \\
\hline MMP-1 & $0.3(0.1,0.9)$ & $0.4(0.1,0.98)$ & $0.2(0.1,0.9)$ \\
\hline \multicolumn{4}{|l|}{ Day 7} \\
\hline ICAM-1 & & $2.4(1.1,5.3)$ & $1.6(0.6,4.2)$ \\
\hline MCP-1 & & $2.3(1.1,5.0)$ & $2.9(1.1,7.3)$ \\
\hline RANTES & & $0.3(0.1,0.7)$ & $0.5(0.1,1.7)$ \\
\hline \multicolumn{4}{|l|}{ Day 14} \\
\hline RANTES & & & $0.3(0.1,0.9)$ \\
\hline ICAM-3 & & & $9.0(3.3,25)$ \\
\hline
\end{tabular}

These are time-oriented logistic risk models that include GA $(23-24,25$ 26 , and $27 \mathrm{wk}$ ).

expect elevated levels to intensify the inflammatory process and increase the likelihood of BPD. In fact, elevated levels of other chemotactic agents, including IL-8 and MIP-1, have been observed in bronchial alveolar lavage fluid in infants who develop BPD (20). However, there is evidence that RANTES actually protects against organ damage in animal models of inflammation-mediated diseases $(21,22)$. In addition, an in vitro study provides support that RANTES reduces inflammation (23). Thus, the association of reduced risk with elevated concentrations of RANTES might reflect antiinflammation and protection.

Other proteins highly associated with both mild/moderate and severe BPD are adhesion molecules, most notably ICAM-1 and ICAM-3. As a group, adhesion molecules promote migration of inflammatory cells from the blood to sites of injury in the lung and their primary site of action is at the blood-tissue interface (24). Because of this site of action, blood levels of adhesion molecules might be elevated in organ-specific inflammation. Therefore, it is not surprising that increased concentrations in blood are also associated with BPD risk. Other circulating inflammatory proteins that are elevated in BPD may not be of pulmonary origin. Rather, their presence may result from a systemic response to injury or inflammation in the lung (e.g. SAA or CRP). It is also possible that under certain circumstances, proteins produced by cells in the lung remain in the lung compartment, whereas under other circumstances (e.g. intense pulmonary inflammation), they
Table 4. ORs (and 99\% CIs) of mild/moderate BPD and severe $B P D$ (vs no BPD) associated with a concentration of the protein on the left in the highest quartile for GA and day blood was obtained relative to the risk among infants whose concentration was in the lower three quartiles

\begin{tabular}{|c|c|c|c|}
\hline Protein & Day 1 & Days 1 and 7 & Days 1,7 , and 14 \\
\hline \multicolumn{4}{|c|}{ Model for mild/moderate BPD } \\
\hline \multicolumn{4}{|l|}{ Day 1} \\
\hline GA $23-24$ & $7.5(4.1,13)$ & $8.3(4.4,16)$ & $9.5(4.7,19)$ \\
\hline GA $25-26$ & $2.5(1.6,4.0)$ & $2.8(1.6,4.4)$ & $2.9(1.7,5.0)$ \\
\hline $\mathrm{BWZ}<-1$ & $3.1(1.8,5.4)$ & $2.8(1.5,4.9)$ & $2.1(1.1,4.0)$ \\
\hline TNF- $\alpha$ & $1.8(1.2,2.9)$ & $1.9(1.2,3.1)$ & $2.0(1.2,3.4)$ \\
\hline \multicolumn{4}{|l|}{ Day 7} \\
\hline ICAM-1 & & $1.9(1.2,3.2)$ & $1.5(0.9,2.7)$ \\
\hline MIP-1 $\beta$ & & $0.5(0.3,0.8)$ & $0.5(0.3,0.9)$ \\
\hline \multicolumn{4}{|l|}{ Day 14} \\
\hline ICAM-1 & & & $2.7(1.5,5.0)$ \\
\hline IL- $1 \beta$ & & & $1.9(1.2,3.2)$ \\
\hline RANTES & & & $0.5(0.3,0.8)$ \\
\hline \multicolumn{4}{|c|}{ Model for severe BPD } \\
\hline \multicolumn{4}{|l|}{ Day 1} \\
\hline GA $23-24$ & $9.9(3.5,28)$ & $13(4.1,39)$ & $18(4.3,78)$ \\
\hline GA $25-26$ & $2.8(1.1,6.8)$ & $2.8(1.1,7.2)$ & $4.9(1.5,16)$ \\
\hline $\mathrm{BWZ}<-1$ & $4.6(2.0,11)$ & $4.3(1.8,11)$ & $4.2(1.4,12)$ \\
\hline VCAM-1 & $2.7(1.2,6.1)$ & $3.7(1.5,9.0)$ & $4.5(1.5,13)$ \\
\hline MMP-1 & $0.3(0.1,0.9)$ & $0.4(0.1,0.98)$ & $0.2(0.1,0.9)$ \\
\hline \multicolumn{4}{|l|}{ Day 7} \\
\hline RANTES & & $0.3(0.1,0.9)$ & $0.6(0.2,2.2)$ \\
\hline MCP-1 & & $2.6(1.2,5.7)$ & $3.2(1.2,8.3)$ \\
\hline \multicolumn{4}{|l|}{ Day 14} \\
\hline ICAM-1 & & & $10(3.8,28)$ \\
\hline RANTES & & & $0.2(0.1,0.8)$ \\
\hline
\end{tabular}

These are time-oriented logistic risk models that include GA $(23-24,25-$ 26 , and $27 \mathrm{wk}$ ) and BWZ score $<-1$.

BWZ, birth weight $Z$ score.

may "leak" into the circulation (25). Because of these uncertainties, we advise caution in drawing inferences about the observed changes in blood proteins and pulmonary pathology.

Some important observations resulted from developing time-oriented risk models for BPD that both included and excluded specific, known clinical risk factors. In one model, we included birth weight $Z$ score, a marker of fetal growth restriction, because it is both a known BPD risk factor $(16,26)$ and is associated with decreased placental expression of selected inflammatory cytokines (27). The associations between protein concentrations and BPD risk in this model were similar to those in the model that excluded birth weight $Z$ score. This finding suggests that the increased BPD risk associated with fetal growth restriction is probably not mediated by circulating inflammation-associated proteins. By contrast, the addition of mechanical ventilation at $7 \mathrm{~d}$ changed the apparent influence of ICAM-1 and RANTES, suggesting that mechanical ventilation may influence pathogenesis by altering expression of these proteins.

This study has a number of strengths. We included a large number of infants, making it unlikely that we missed important associations because of the lack of statistical power, and we collected all of our data prospectively. We selected infants based on GA, not birth weight, to minimize confounding because of factors related to fetal growth restriction, and with regression models, we adjusted for fetal growth restriction. 
Table 5. ORs (and 99\% CIs) of mild/moderate BPD and severe $B P D$ (vs no BPD) of the protein on the left associated with a concentration of the protein on the left in the highest quartile for GA and day blood was obtained relative to the risk among infants whose concentration was in the lower three quartiles

\begin{tabular}{|c|c|c|c|}
\hline Protein & Day 1 & Days 1 and 7 & Days 1,7 , and 14 \\
\hline \multicolumn{4}{|c|}{ Model for mild/moderate BPD } \\
\hline \multicolumn{4}{|l|}{ Day 1} \\
\hline GA $23-24$ & $7.5(4.1,13)$ & $4.1(2.1,8.3)$ & $5.6(2.6,12)$ \\
\hline GA $25-26$ & $2.5(1.6,4.0)$ & $1.9(1.1,3.2)$ & $2.1(1.2,3.8)$ \\
\hline $\mathrm{BWZ}<-1$ & $3.1(1.8,5.4)$ & $2.4(1.3,4.5)$ & $2.0(1.02,3.9)$ \\
\hline TNF- $\alpha$ & $1.8(1.2,2.9)$ & $2.1(1.3,3.5)$ & $2.0(1.2,3.5)$ \\
\hline \multicolumn{4}{|l|}{ Day 7} \\
\hline $\begin{array}{l}\text { Mechanical } \\
\text { ventilation }\end{array}$ & & $4.2(2.6,6.9)$ & $2.9(1.7,5.1)$ \\
\hline TNF-R2 & & $2.2(1.3,3.9)$ & $2.1(1.1,3.9)$ \\
\hline SAA & & $0.5(0.3,0.9)$ & $0.5(0.3,0.9)$ \\
\hline MIP-1 $\beta$ & & $0.5(0.3,0.9)$ & $0.6(0.3,1.02)$ \\
\hline \multicolumn{4}{|l|}{ Day 14} \\
\hline IL- $1 \beta$ & & & $1.9(1.1,3.2)$ \\
\hline ICAM-3 & & & $2.4(1.3,4.4)$ \\
\hline \multicolumn{4}{|c|}{ Model for severe BPD } \\
\hline \multicolumn{4}{|l|}{ Day 1} \\
\hline GA $23-24$ & $9.9(3.5,28)$ & $4.3(1.3,13)$ & $5.0(1.3,19)$ \\
\hline GA $25-26$ & $2.8(1.1,6.8)$ & $1.8(0.7,4.8)$ & $2.4(0.9,7.6)$ \\
\hline $\mathrm{BWZ}<-1$ & $4.6(2.0,11)$ & $3.8(1.5,9.4)$ & $3.0(1.1,8.1)$ \\
\hline VCAM-1 & $2.7(1.2,6.1)$ & $4.1(1.6,11)$ & $4.0(1.4,12)$ \\
\hline MMP-1 & $0.3(0.1,0.9)$ & $0.4(0.1,0.98)$ & $0.2(0.1,0.9)$ \\
\hline \multicolumn{4}{|l|}{ Day 7} \\
\hline $\begin{array}{l}\text { Mechanical } \\
\text { ventilation }\end{array}$ & & $15(4.2,56)$ & $11(2.7,50)$ \\
\hline \multicolumn{4}{|l|}{ Day 14} \\
\hline ICAM-3 & & & $5.6(2.2,14)$ \\
\hline
\end{tabular}

These are time-oriented logistic risk models that include GA (23-24, 25-26, and $27 \mathrm{wk}), \mathrm{BWZ}$ score $<-1$ and mechanical ventilation on $\mathrm{d} 7$. BWZ, birth weight $Z$ score.

We did not include infants who died before 36 wk PMA, thereby restricting this to a study of BPD and not the composite outcome of BPD or death.

This study also has limitations. For practical reasons, our cohort was limited to infants who had complete neurodevelopmental evaluation at $24 \mathrm{mo}$. This resulted in the nonrandom exclusion of two potentially important cohorts. The incidence of both mild/moderate and severe BPD in the cohort of infants who survived to 24 mo but for whom protein measurements were not available was only slightly lower than in our study cohort. Therefore, their exclusion is not likely to have resulted in a significant bias in the results. The incidence of severe BPD among infants who died between 36 wk PMA and 24 mo was significantly higher (47\%) than in our study cohort (9\%). However, the effect of exclusion of these infants is likely to be minimal because they represent a small proportion of the ELGAN Study cohort $(\approx 4 \%)$. The diagnosis of BPD was made on the basis of clinicians' decisions to treat with mechanical ventilation or supplemental oxygen, not on the basis of physiologic disturbances, and clinical practices almost certainly varied among centers. Finally, because this was an observational study, we cannot know whether the protein elevations were associated with BPD in a cause and effect relationship or whether the elevations are surrogates for other processes that might have influenced BPD risk.
Our study has several implications for researchers interested in preventing BPD. First, the different risk factor profile for mild/moderate and severe BPD suggest that BPD is a heterogeneous condition. Consideration of this heterogeneity could lead to more informative epidemiologic studies. Second, our findings suggest the possibility that inflammation-associated proteins in neonatal blood could serve as biomarkers of modifiable biological processes involved in the development of BPD. If so, these biomarkers could be used as response measures in intervention studies. Third, at least some aspect of the fetal and neonatal inflammatory response is associated with a lower risk of BPD. Thus, one approach to prevention might be the enhancement of endogenous protectors.

Acknowledgments. We thank our ELGAN Study colleagues: Bhavesh L. Shah, Baystate Medical Center, Springfield, MA; Camilia Martin, Beth Israel Deaconess Medical Center, Boston, MA; Linda Van Marter, Brigham \& Women's Hospital, Boston, MA; Robert Insoft, MA General Hospital, Boston, MA; Karl Kuban, Boston Medical Center, Boston, MA; Francis Bednarek, U Mass Memorial Health Center, Worcester, MA; Olaf Dammann, John Fiascone, and Cynthia Cole, Tufts Medical Center, Boston, MA; Richard A. Ehrenkranz, Yale-New Haven Children's Hospital, New Haven CT; T. Michael O'Shea, Wake Forest University/Baptist Medical Center, Winston-Salem, NC; Stephen C. Engelke, University Health Systems of Eastern Carolina, Greenville, NC; Carl Bose, University of North Carolina, Chapel Hill, NC; Mariel Poortenga, DeVos Children's Hospital, Grand Rapids, MI; Padima Karna, Sparrow Hospital, Lansing, MI; Nigel Paneth, MI State University, East Lansing, MI; Michael D. Schreiber, University of Chicago Hospital, Chicago, IL; Daniel Batton, William Beaumont Hospital, Royal Oak, MI; Greg Pavlov, Frontier Science and Technology Research Foundation, Amherst, NY; the technical team at Dr. Fichorova's Lab, Brigham \& Women's Hospital, Hidemi Yamamoto, Vanessa Tang, Yoshika Ymamoto, Huaiping Yuan, Rita Sassi; and project officer, Deborah Hirtz.

\section{REFERENCES}

1. Merritt TA, Cochrane CG, Holcomb K, Bohl B, Hallman M, Strayer D, Edwards DK III, Gluck L 1983 Elastase and alpha 1-proteinase inhibitor activity in tracheal aspirates during respiratory distress syndrome. Role of inflammation in the pathogenesis of bronchopulmonary dysplasia. J Clin Invest 72:656-666

2. Groneck P, Gotze-Speer B, Oppermann M, Eiffert H, Speer CP 1994 Association of pulmonary inflammation and increased microvascular permeability during the development of bronchopulmonary dysplasia: a sequential analysis of inflammatory mediators in respiratory fluids of high-risk preterm neonates. Pediatrics 93:712-718

3. Ryan RM, Ahmed Q, Lakshminrusimha S 2008 Inflammatory mediators in the immunobiology of bronchopulmonary dysplasia. Clin Rev Allergy Immunol 34:174-190

4. Groneck P, Speer CP 1995 Inflammatory mediators and bronchopulmonary dysplasia. Arch Dis Child Fetal Neonatal Ed 73:F1-F3

5. Tremblay L, Valenza F, Ribeiro SP, Li J, Slutsky AS 1997 Injurious ventilatory strategies increase cytokines and c-fos m-RNA expression in an isolated rat lung model. J Clin Invest 99:944-952

6. Bhandari V, Elias JA 2006 Cytokines in tolerance to hyperoxia-induced injury in the developing and adult lung. Free Radic Biol Med 41:4-18

7. Ambalavanan N, Carlo WA, D'Angio CT, McDonald SA, Das A, Schendel D, Thorsen P, Higgins RD 2009 Cytokines associated with bronchopulmonary dysplasia or death in extremely low birth weight infants. Pediatrics 123:1132-1141

8. Paananen R, Husa AK, Vuolteenaho R, Herva R, Kaukola T, Hallman M 2009 Blood cytokines during the perinatal period in very preterm infants: relationship of 
inflammatory response and bronchopulmonary dysplasia. J Pediatr 154:39.e343.e33

9. O'Shea TM, Allred EN, Dammann O, Hirtz D, Kuban KC, Paneth N, Leviton A 2009 The ELGAN study of the brain and related disorders in extremely low gestational age newborns. Early Hum Dev 85:719-725

10. Yudkin PL, Aboualfa M, Eyre JA, Redman CW, Wilkinson AR 1987 New birthweight and head circumference centiles for gestational ages 24 to 42 weeks. Early Hum Dev 15:45-52

11. Hecht JL, Allred EN, Kliman HJ, Zambrano E, Doss BJ, Husain A, Pflueger SM, Chang CH, Livasy CA, Roberts D, Bhan I, Ross DW, Senagore PK, Leviton A 2008 Histological characteristics of singleton placentas delivered before the 28th week of gestation. Pathology 40:372-376

12. Fichorova RN, Richardson-Harman N, Alfano M, Belec L, Carbonneil C, Chen S Cosentino L, Curtis K, Dezzutti CS, Donoval B, Doncel GF, Donaghay M, Grive JC, Guzman E, Hayes M, Herold B, Hillier S, Lackman-Smith C, Landay A Margolis L, Mayer KH, Pasicznyk JM, Pallansch-Cokonis M, Poli G, Reichelderfer P, Roberts P, Rodriguez I, Saidi H, Sassi RR, Shattock R, Cummins JE Jr 2008 Biological and technical variables affecting immunoassay recovery of cytokines from human serum and simulated vaginal fluid: a multicenter study. Anal Chem 80:4741-4751

13. Dubin N, Pasternack BS 1986 Risk assessment for case-control subgroups by polychotomous logistic regression. Am J Epidemiol 123:1101-1117

14. Lucey DR, Clerici M, Shearer GM 1996 Type 1 and type 2 cytokine dysregulation in human infectious, neoplastic, and inflammatory diseases. Clin Microbiol Rev 9:532-562

15. Viscardi RM, Muhumuza CK, Rodriguez A, Fairchild KD, Sun CC, Gross GW, Campbell AB, Wilson PD, Hester L, Hasday JD 2004 Inflammatory markers in intrauterine and fetal blood and cerebrospinal fluid compartments are associated with adverse pulmonary and neurologic outcomes in preterm infants. Pediatr Res 55:1009-1017

16. Bose C, Van Marter LJ, Laughon M, O'Shea TM, Allred EN, Karna P, Ehrenkranz RA, Boggess K, Leviton A 2009 Fetal growth restriction and chronic lung disease among infants born before the 28th week of gestation. Pediatrics 124:e450-e458
17. Bohrer B, Silveira RC, Neto EC, Procianoy RS 2010 Mechanical ventilation of newborns infant changes in plasma pro- and anti-inflammatory cytokines. J Pediatr $156: 16-19$

18. Polglase GR, Hillman NH, Pillow JJ, Cheah FC, Nitsos I, Moss TJ, Kramer BW, Ikegami M, Kallapur SG, Jobe AH 2008 Positive end-expiratory pressure and tidal volume during initial ventilation of preterm lambs. Pediatr Res 64:517-522

19. Charo IF, Ransohoff RM 2006 The many roles of chemokines and chemokine receptors in inflammation. N Engl J Med 354:610-621

20. Baier RJ, Loggins J, Kruger TE 2001 Monocyte chemoattractant protein-1 and interleukin-8 are increased in bronchopulmonary dysplasia: relation to isolation of Ureaplasma urealyticum. J Investig Med 49:362-369

21. Shahrara S, Proudfoot AE, Woods JM, Ruth JH, Amin MA, Park CC, Haas CS, Pope RM, Haines GK, Zha YY, Koch AE 2005 Amelioration of rat adjuvant-induced arthritis by Met-RANTES. Arthritis Rheum 52:1907-1919

22. Dobaczewski M, Xia Y, Bujak M, Gonzalez-Quesada C, Frangogiannis NG 2010 CCR5 signaling suppresses inflammation and reduces adverse remodeling of the infarcted heart, mediating recruitment of regulatory T cells. Am J Pathol 176:21772187

23. Shahrara S, Park CC, Temkin V, Jarvis JW, Volin MV, Pope RM 2006 RANTES modulates TLR4-induced cytokine secretion in human peripheral blood monocytes. J Immunol 177:5077-5087

24. Liles WC, Van Voorhis WC 1995 Review: nomenclature and biologic significance of cytokines involved in inflammation and the host immune response. J Infect Dis 172:1573-1580

25. Plötz FB, Slutsky AS, van Vught AJ, Heijnen CJ 2004 Ventilator-induced lung injury and multiple system organ failure: a critical review of facts and hypotheses Intensive Care Med 30:1865-1872

26. Garite TJ, Clark R, Thorp JA 2004 Intrauterine growth restriction increases morbidity and mortality among premature neonates. Am J Obstet Gynecol 191:481-487

27. Hahn-Zoric M, Hagberg H, Kjellmer I, Ellis J, Wennergren M, Hanson LA 2002 Aberrations in placental cytokine mRNA related to intrauterine growth retardation. Pediatr Res 51:201-206 